\title{
SYNTHESIS OF SOME THIAZOLIDINE DERIVATIVES OF 1,4-BENZOQUINONE AS POTENTIAL ANTIMICROBIAL AGENTS
}

\author{
I. Chaaban ${ }^{1}$, A. A. Bekhit ${ }^{1}$ and Elsayed Aboulmagd ${ }^{2}$ \\ ${ }^{1}$ Department of Pharmaceutical Chemistry, ${ }^{2}$ Department of Microbiology, Faculty of \\ Pharmacy, University of Alexandria, Alexandria 21521, Egypt
}

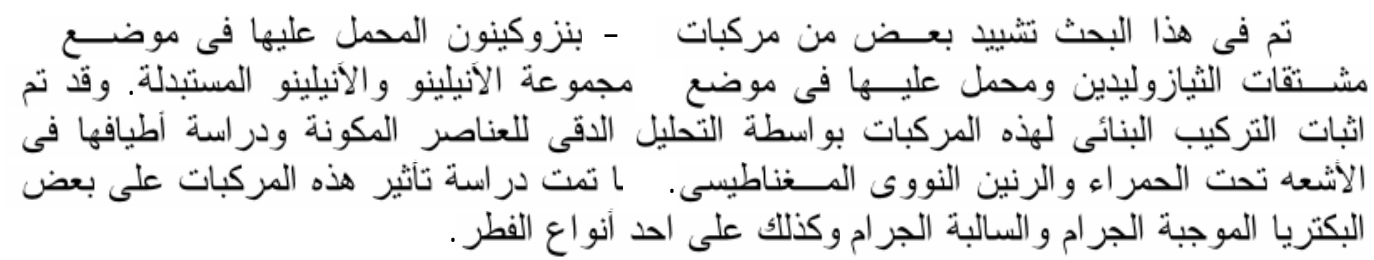

A series of 2,5-disubstituted-1,4-benzoquinone derivatives were synthesized and evaluated for their antimicrobial activity. Coupling of 2,5-dihydroxybenzoic acid (gentisic acid) with the selected thiazolidine derivative using EDC and HOBt afforded the corresponding (4R)-3-(2,5dihydroxybenzoyl)-5,5-dimethyl-N-substituted benzylthiazolidine-4-carboxamide $2 a-d$. These compounds were subsequently oxidized with ferric chloride to afford the corresponding 1,4benzoquinones 3a-d. The reaction between 1,4-benzoquinone derivatives and the appropriate amine resulted in the targeted 2,5-disubstituted-1,4-benzoquinone derivatives 4a-l. The structure of the newly synthesized compound were confirmed by elemental microanalyses, IR and ${ }^{1} H$ NMR spectra. The antimicrobial activity of target compounds $4 a-l$ was performed against Escherichia coli (E. coli) ATCC 25922 as Gram-negative bacteria, Staphylococcus aureus (S. aureus) ATCC 19433 as Gram-positive bacteria and Candida albicans (C. albicans) as yeast like fungi were determined.

\section{INTRODUCTION}

The evergrowing clinical importance of developing drug-resistant bacterial pathogens has lent additional urgency to microbiological and antibacterial research. ${ }^{1}$ In addition, in recent years, fungal infection became an important complication and a major cause of morbidity and mortality in immunocompromised individuals such as those suffering from tuberculosis, cancer or AIDS and in organ transplant cases. ${ }^{2,3}$ Within a program aimed at developing new medicinal agents related to 1,4-benzoquinone derivatives, some pyrazolyl, oxadiazolyl or thiadiazolyl derivatives of 1,4-benzoquinone were synthesized in our laboratory and evaluated for their antimicrobial effects. ${ }^{4-7}$ Moreover, thiazolidine derivatives have been reported to exhibit antimicrobial activity. ${ }^{8}$ In addition and as a continuation of our continuous related program, 1,4-benzoquinone derivatives were synthesized having within their structure the thiazolidine function attached to position 2 through a carbonyl linker and 4-substituted anilino moiety attached to position 5 , in order to investigate the effect of the thiazolidine moiety on the antimicrobial activity of the 1,4benzoquinone derivatives.

\section{EXPERINEMTAL}

Melting points were uncorrected and determined in open glass capillaries using a Thomas capillary melting point apparatus. IR spectra were recorded on 470-Shimadzu infrared spectrophotometer. ${ }^{1} \mathrm{H}-\mathrm{NMR}$ spectra were obtained from a Varian XL-200 $\mathrm{MHz}$ Spectrometer, and the chemical shifts are given in $\delta(\mathrm{ppm})$ down field from tetramethylsilane (TMS) as an internal standard. Elemental analyses were performed at the Microanalytical Unit, Faculty of Science, Cairo University, Cairo, Egypt. 
Synthesis of the compounds

\section{(4R)-3-(2,5-Dihydroxybenzoyl)-5,5-di- methyl-N-substituted benzylthiazolidine-4- carboxamide 2a-d}

To a solution of the appropriate N-Boc thiazolidine derivatives ${ }^{9}$ 1a-d (5 mmol), in (20 $\mathrm{ml}) 4 \mathrm{~N} \mathrm{HCl} /$ Dioxane, at $0^{\circ}$ anisole $(1.9 \mathrm{ml}, 10$ $\mathrm{mmol}$ ) was added and stirred for $2 \mathrm{~h}$ at room temperature. The solvent was evaporated in vacuo and ether $(10 \mathrm{ml})$ was added. The mixture was centrifuged, and the residue was dissolved in dimethylformamide (DMF) (15 $\mathrm{ml})$. To the above solution, were added at $0^{\circ}$ gentisic acid (693 $\mathrm{mg}, 4.5 \mathrm{mmol}$ ), hydrated hydroxybenzotriazole $\left(\mathrm{HOBt} . \mathrm{H}_{2} \mathrm{O}\right)(766 \mathrm{mg}, 5$ mmol), 1-ethyl-3-(3-dimethyl aminopropyl) carbodiimide hydrochloride (EDC.HCl) (1.05 $\mathrm{g}, 5.5 \mathrm{mmol})$ and triethylamine $\left(\mathrm{Et}_{3} \mathrm{~N}\right)(1.39 \mathrm{ml}$, $10 \mathrm{mmol})$. The mixture was stirred for an overnight at room temperature. The solvent was then removed under vacuum and the residue was extracted with ethyl acetate (AcOEt). The combined organic extracts were successively washed with $10 \%$ citric acid, $5 \%$ $\mathrm{NaHCO}_{3}$ and finally with brine. The extracts were then dried over anhydrous $\mathrm{Na}_{2} \mathrm{SO}_{4}$, filtered, and evaporated under reduced pressure. The residues were crystallized from AcOEt / n-hexane, Table 1.

IR $\left(\mathrm{KBr}, \mathrm{cm}^{-1}\right)(\mathbf{2 a - d}): 3332-3325(\mathrm{OH})$, 3140-3134 (NH), 1678-1673 (C=O), 1668-1665 $(\mathrm{C}=\mathrm{O})$.

${ }^{1} \mathrm{HNMR}(\mathbf{2 a})$ : (DMSO-d $\left.\mathrm{d}_{6}, \delta, \mathrm{ppm}\right): 1.42(\mathrm{~s}$, $3 \mathrm{H}$, thiazol- $\left.\mathrm{C}_{5}-\mathrm{CH}_{3}\right), 1.46\left(\mathrm{~s}, 3 \mathrm{H}\right.$, thiazol- $\mathrm{C}_{5^{-}}$ $\left.\mathrm{CH}_{3}\right), 4.03\left(\mathrm{~d}, \mathrm{~J}=17.7 \mathrm{~Hz}, 1 \mathrm{H}\right.$, thiazol- $\left.\mathrm{C}_{4}-\mathrm{H}\right)$, $4.24\left(\mathrm{~m}, 2 \mathrm{H}\right.$, thiazol- $\left.\mathrm{CH}_{2}\right), 4.53(\mathrm{~s}, 2 \mathrm{H}$, $\mathrm{CH}_{2} \mathrm{NH}$ ), 6.74-7.22 (m, 8H, Ar-H), 8.48 (br s, $1 \mathrm{H}, \mathrm{NH}, \mathrm{D}_{2} \mathrm{O}$ exchangeable), $10.32(\mathrm{~s}, 1 \mathrm{H}, \mathrm{OH}$, $\mathrm{D}_{2} \mathrm{O}$ exchangeable), $12.94\left(\mathrm{~s}, 1 \mathrm{H}, \mathrm{OH}, \mathrm{D}_{2} \mathrm{O}\right.$ exchangeable).

${ }^{1} \mathrm{HNMR}$ (2b): (DMSO-d $\left.\mathrm{d}_{6}, \delta, \mathrm{ppm}\right): 1.44$ (s, 3H, thiazol- $\left.\mathrm{C}_{5}-\mathrm{CH}_{3}\right), 1.51\left(\mathrm{~s}, 3 \mathrm{H}\right.$, thiazol- $\mathrm{C}_{5^{-}}$ $\left.\mathrm{CH}_{3}\right), 4.09\left(\mathrm{~d}, \mathrm{~J}=17.7 \mathrm{~Hz}, 1 \mathrm{H}\right.$, thiazol- $\left.\mathrm{C}_{4}-\mathrm{H}\right)$, $4.28\left(\mathrm{~m}, 2 \mathrm{H}\right.$, thiazol- $\left.\mathrm{CH}_{2}\right), 4.54(\mathrm{~s}, 2 \mathrm{H}$, $\mathrm{CH}_{2} \mathrm{NH}$ ), 6.71-7.27 (m, 7H, Ar-H), 8.45 (br s, $1 \mathrm{H}, \mathrm{NH}, \mathrm{D}_{2} \mathrm{O}$ exchangeable), $10.29(\mathrm{~s}, 1 \mathrm{H}, \mathrm{OH}$, $\mathrm{D}_{2} \mathrm{O}$ exchangeable), $12.85\left(\mathrm{~s}, 1 \mathrm{H}, \mathrm{OH}, \mathrm{D}_{2} \mathrm{O}\right.$ exchangeable).

${ }^{1} \mathrm{HNMR}$ (2c): (DMSO-d $\left.\mathrm{d}_{6}, \delta, \mathrm{ppm}\right): 1.42(\mathrm{~s}$, $3 \mathrm{H}$, thiazol- $\left.\mathrm{C}_{5}-\mathrm{CH}_{3}\right), 1.56\left(\mathrm{~s}, 3 \mathrm{H}\right.$, thiazol- $\mathrm{C}_{5^{-}}$ $\left.\mathrm{CH}_{3}\right), 4.11\left(\mathrm{~d}, \mathrm{~J}=17.7 \mathrm{~Hz}, 1 \mathrm{H}\right.$, thiazol- $\left.\mathrm{C}_{4}-\mathrm{H}\right)$, $4.31\left(\mathrm{~m}, 2 \mathrm{H}\right.$, thiazol- $\left.\mathrm{CH}_{2}\right), 4.47(\mathrm{~s}, 2 \mathrm{H}$, $\mathrm{CH}_{2} \mathrm{NH}$ ), 6.71-7.28 (m, 7H, Ar-H), 8.42 (br s,
$1 \mathrm{H}, \mathrm{NH}, \mathrm{D}_{2} \mathrm{O}$ exchangeable), $10.31(\mathrm{~s}, 1 \mathrm{H}, \mathrm{OH}$, $\mathrm{D}_{2} \mathrm{O}$ exchangeable), $12.87\left(\mathrm{~s}, 1 \mathrm{H}, \mathrm{OH}, \mathrm{D}_{2} \mathrm{O}\right.$ exchangeable).

${ }^{1} \mathrm{HNMR}$ (2d): (DMSO-d $\left.6, \delta, \mathrm{ppm}\right): 1.47$ (s, 3H, thiazol- $\left.\mathrm{C}_{5}-\mathrm{CH}_{3}\right), 1.54\left(\mathrm{~s}, 3 \mathrm{H}\right.$, thiazol- $\mathrm{C}_{5^{-}}$ $\left.\mathrm{CH}_{3}\right), 4.12\left(\mathrm{~d}, \mathrm{~J}=17.7 \mathrm{~Hz}, 1 \mathrm{H}\right.$, thiazol- $\left.\mathrm{C}_{4}-\mathrm{H}\right)$, $4.33\left(\mathrm{~m}, 2 \mathrm{H}\right.$, thiazol- $\left.\mathrm{CH}_{2}\right), 4.44(\mathrm{~s}, 2 \mathrm{H}$, $\mathrm{CH}_{2} \mathrm{NH}$ ), 6.73-7.32 (m, 7H, Ar-H), 8.46 (br s, $1 \mathrm{H}, \mathrm{NH}, \mathrm{D}_{2} \mathrm{O}$ exchangeable), $10.34(\mathrm{~s}, 1 \mathrm{H}, \mathrm{OH}$, $\mathrm{D}_{2} \mathrm{O}$ exchangeable $), 12.91\left(\mathrm{~s}, 1 \mathrm{H}, \mathrm{OH}, \mathrm{D}_{2} \mathrm{O}\right.$ exchangeable).

\section{(4R)-3-[(3,6-Dioxo-1,4-cyclohexadienyl) carbonyl]-5,5-dimethyl-N-substituted benzylthiazolidine-4-carboxamide 3a-d}

To a solution of the appropriate hydroquinone 2a-d $(5 \mathrm{mmol})$ in dimethylformamide (DMF) (10 ml), a solution of ferric chloride $(10 \%, 20 \mathrm{ml})$ was portionwise added with stirring for $15 \mathrm{~min} .{ }^{4}$ The reaction mixture was diluted with water $(50 \mathrm{ml})$, whereupon orange precipitates were formed. The solid products were filtered, washed with water till free from ferric ion, dried and recrystallized from aqueous dimethylformamide. Table 1.

IR $\left(\mathrm{KBr}, \mathrm{cm}^{-1}\right)(\mathbf{3 a - d}): 3154-3148(\mathrm{NH})$, 1680-1675 (C=O), 1671-1665 $(\mathrm{C}=\mathrm{O})$.

${ }^{1} \mathrm{HNMR}$ (3a): (DMSO-d 6 , $\left.\delta, \mathrm{ppm}\right): 1.46$ (s, $3 \mathrm{H}$, thiazol- $\left.\mathrm{C}_{5}-\mathrm{CH}_{3}\right), 1.51\left(\mathrm{~s}, 3 \mathrm{H}\right.$, thiazol- $\mathrm{C}_{5^{-}}$ $\left.\mathrm{CH}_{3}\right), 4.07\left(\mathrm{~d}, \mathrm{~J}=17.7 \mathrm{~Hz}, 1 \mathrm{H}\right.$, thiazol- $\left.\mathrm{C}_{4}-\mathrm{H}\right)$, $4.28\left(\mathrm{~m}, 2 \mathrm{H}\right.$, thiazol- $\left.\mathrm{CH}_{2}\right), 4.51(\mathrm{~s}, 2 \mathrm{H}$, $\mathrm{CH}_{2} \mathrm{NH}$ ), 6.78-7.64 (m, 8H, Ar-H), 8.52 (br s, $1 \mathrm{H}, \mathrm{NH}, \mathrm{D}_{2} \mathrm{O}$ exchangeable).

${ }^{1} \mathrm{HNMR}$ (3b): (DMSO-d $\left.6, \delta, \mathrm{ppm}\right): 1.40$ (s, $3 \mathrm{H}$, thiazol- $\left.\mathrm{C}_{5}-\mathrm{CH}_{3}\right), 1.46\left(\mathrm{~s}, 3 \mathrm{H}\right.$, thiazol- $\mathrm{C}_{5^{-}}$ $\left.\mathrm{CH}_{3}\right), 4.10\left(\mathrm{~d}, \mathrm{~J}=17.7 \mathrm{~Hz}, 1 \mathrm{H}\right.$, thiazol- $\left.\mathrm{C}_{4}-\mathrm{H}\right)$, $4.32\left(\mathrm{~m}, 2 \mathrm{H}\right.$, thiazol- $\left.\mathrm{CH}_{2}\right), 4.52(\mathrm{~s}, 2 \mathrm{H}$, $\mathrm{CH}_{2} \mathrm{NH}$ ), 6.74-7.63 (m, 7H, Ar-H), 8.47 (br s, $1 \mathrm{H}, \mathrm{NH}, \mathrm{D}_{2} \mathrm{O}$ exchangeable).

${ }^{1} \mathrm{HNMR}(3 \mathrm{c})$ : (DMSO-d $\left.\mathrm{d}_{6}, \delta, \mathrm{ppm}\right): 1.41$ (s, $3 \mathrm{H}$, thiazol- $\left.\mathrm{C}_{5}-\mathrm{CH}_{3}\right), 1.52\left(\mathrm{~s}, 3 \mathrm{H}\right.$, thiazol- $\mathrm{C}_{5^{-}}$ $\left.\mathrm{CH}_{3}\right), 4.08\left(\mathrm{~d}, \mathrm{~J}=17.7 \mathrm{~Hz}, 1 \mathrm{H}\right.$, thiazol- $\left.\mathrm{C}_{4}-\mathrm{H}\right)$, $4.33\left(\mathrm{~m}, 2 \mathrm{H}\right.$, thiazol- $\left.\mathrm{CH}_{2}\right), 4.46(\mathrm{~s}, 2 \mathrm{H}$, $\mathrm{CH}_{2} \mathrm{NH}$ ), 6.72-7.68 (m, 7H, Ar-H), 8.53 (br s, $1 \mathrm{H}, \mathrm{NH}, \mathrm{D}_{2} \mathrm{O}$ exchangeable).

${ }^{1} \mathrm{HNMR}$ (3d): (DMSO-d $\left.\mathrm{d}_{6}, \delta, \mathrm{ppm}\right): 1.44$ (s, 3H, thiazol- $\left.\mathrm{C}_{5^{-}}-\mathrm{CH}_{3}\right), 1.52\left(\mathrm{~s}, 3 \mathrm{H}\right.$, thiazol- $\mathrm{C}_{5^{-}}$ $\left.\mathrm{CH}_{3}\right), 4.11\left(\mathrm{~d}, \mathrm{~J}=17.7 \mathrm{~Hz}, 1 \mathrm{H}\right.$, thiazol- $\left.\mathrm{C}_{4}-\mathrm{H}\right)$, $4.34\left(\mathrm{~m}, 2 \mathrm{H}\right.$, thiazol- $\left.\mathrm{CH}_{2}\right), 4.47(\mathrm{~s}, 2 \mathrm{H}$, $\mathrm{CH}_{2} \mathrm{NH}$ ), 6.71-7.67 (m, 7H, Ar-H), 8.54 (br s, $1 \mathrm{H}, \mathrm{NH}, \mathrm{D}_{2} \mathrm{O}$ exchangeable). 
Table 1: Physical and analytical data of compounds 2a-d and 3a-d.

\begin{tabular}{|c|c|c|c|c|c|c|c|c|c|}
\hline \multirow{2}{*}{$\begin{array}{c}\text { Comp. } \\
\text { No. }\end{array}$} & \multirow[t]{2}{*}{$\mathrm{R}^{1}$} & \multirow{2}{*}{$\begin{array}{l}\text { Yield } \\
(\%)\end{array}$} & \multirow[t]{2}{*}{$\underset{\mathrm{o}}{\mathrm{Mp}}$} & \multirow{2}{*}{$\begin{array}{l}\text { Mol. Formula } \\
\text { (Mol. wt.) }\end{array}$} & \multicolumn{5}{|c|}{$\begin{array}{l}\text { Analyses (\%) } \\
\text { Calcd/Found }\end{array}$} \\
\hline & & & & & $\mathrm{C}$ & $\mathrm{H}$ & $F$ & $\mathrm{~N}$ & $S$ \\
\hline $2 a$ & $\mathrm{H}$ & 82 & $256-7$ & $\begin{array}{c}\mathrm{C}_{20} \mathrm{H}_{22} \mathrm{~N}_{2} \mathrm{O}_{4} \mathrm{~S} \\
(386.46)\end{array}$ & $\begin{array}{l}62.16 \\
62.1\end{array}$ & $\begin{array}{c}5.74 \\
5.5\end{array}$ & ----- & $\begin{array}{l}7.25 \\
7.5\end{array}$ & $\begin{array}{c}8.30 \\
8.4\end{array}$ \\
\hline $2 \mathrm{~b}$ & $2-\mathrm{F}$ & 78 & $264-5$ & $\begin{array}{c}\mathrm{C}_{20} \mathrm{H}_{21} \mathrm{FN}_{2} \mathrm{O}_{4} \mathrm{~S} \\
(404.45)\end{array}$ & $\begin{array}{c}59.39 \\
59.7\end{array}$ & $\begin{array}{c}5.23 \\
5.5\end{array}$ & $\begin{array}{c}4.70 \\
4.3\end{array}$ & $\begin{array}{c}6.93 \\
6.6\end{array}$ & $\begin{array}{l}7.93 \\
7.6\end{array}$ \\
\hline $2 c$ & $3-\mathrm{F}$ & 74 & $267-8$ & $\begin{array}{c}\mathrm{C}_{20} \mathrm{H}_{21} \mathrm{FN}_{2} \mathrm{O}_{4} \mathrm{~S} \\
(404.45)\end{array}$ & $\begin{array}{c}59.39 \\
59.5\end{array}$ & $\begin{array}{c}5.23 \\
5.3\end{array}$ & $\begin{array}{c}4.70 \\
4.9\end{array}$ & $\begin{array}{c}6.93 \\
7.1\end{array}$ & $\begin{array}{l}7.93 \\
7.4\end{array}$ \\
\hline $2 d$ & $4-F$ & 80 & $263-4$ & $\begin{array}{c}\mathrm{C}_{20} \mathrm{H}_{21} \mathrm{FN}_{2} \mathrm{O}_{4} \mathrm{~S} \\
(404.45)\end{array}$ & $\begin{array}{c}59.39 \\
59.2\end{array}$ & $\begin{array}{c}5.23 \\
5.6\end{array}$ & $\begin{array}{r}4.70 \\
4.4\end{array}$ & $\begin{array}{c}6.93 \\
7.0\end{array}$ & $\begin{array}{l}7.93 \\
8.2\end{array}$ \\
\hline $\mathbf{3 a}$ & $\mathrm{H}$ & 72 & decomp. & $\begin{array}{c}\mathrm{C}_{20} \mathrm{H}_{20} \mathrm{~N}_{2} \mathrm{O}_{4} \mathrm{~S} \\
(384.45)\end{array}$ & $\begin{array}{l}62.48 \\
62.5\end{array}$ & $\begin{array}{c}5.24 \\
5.4\end{array}$ & ----- & $\begin{array}{l}7.29 \\
7.6\end{array}$ & $\begin{array}{c}8.34 \\
8.5\end{array}$ \\
\hline $3 b$ & $2-F$ & 79 & decomp. & $\begin{array}{c}\mathrm{C}_{20} \mathrm{H}_{19} \mathrm{FN}_{2} \mathrm{O}_{4} \mathrm{~S} \\
(402.44)\end{array}$ & $\begin{array}{c}59.69 \\
59.7\end{array}$ & $\begin{array}{c}4.76 \\
4.5\end{array}$ & $\begin{array}{c}4.72 \\
4.9\end{array}$ & $\begin{array}{c}6.96 \\
7.9\end{array}$ & $\begin{array}{c}7.97 \\
8.0\end{array}$ \\
\hline $3 c$ & $3-\mathrm{F}$ & 83 & decomp. & $\begin{array}{c}\mathrm{C}_{20} \mathrm{H}_{19} \mathrm{FN}_{2} \mathrm{O}_{4} \mathrm{~S} \\
(402.44)\end{array}$ & $\begin{array}{c}59.69 \\
59.3\end{array}$ & $\begin{array}{c}4.76 \\
4.4\end{array}$ & $\begin{array}{c}4.72 \\
4.3\end{array}$ & $\begin{array}{c}6.96 \\
7.5\end{array}$ & $\begin{array}{c}7.97 \\
8.2\end{array}$ \\
\hline $3 d$ & $4-F$ & 86 & decomp. & $\begin{array}{c}\mathrm{C}_{20} \mathrm{H}_{19} \mathrm{FN}_{2} \mathrm{O}_{4} \mathrm{~S} \\
(402.44) \\
\end{array}$ & $\begin{array}{c}59.69 \\
59.4 \\
\end{array}$ & $\begin{array}{c}4.76 \\
4.8 \\
\end{array}$ & $\begin{array}{r}4.72 \\
4.5 \\
\end{array}$ & $\begin{array}{c}6.96 \\
7.7 \\
\end{array}$ & $\begin{array}{l}7.97 \\
7.7 \\
\end{array}$ \\
\hline
\end{tabular}

(4R)-3-(5-Substituted anilino-[(3,6-dioxo1,4-cyclohexadienyl)carbonyl]-5,5-dimethyl$\mathrm{N}$-substituted benzylthiazolidine-4carboxamide 4a-I

To a solution of the appropriate benzoquinone 3a-d (1 mmol) and aniline or substituted aniline $(0.5 \mathrm{mmol})$ in ethanol $(15$ ml) was added 5 drops of glacial acetic acid. ${ }^{10-}$

${ }^{12}$ The mixture was heated under reflux for 0.5 $\mathrm{h}$ and filtered while hot. The precipitates were washed with $5 \mathrm{ml}$ boiling ethanol, dried and crystallized from aqueous dimethylformamide, Table 2. All compounds decompose before melting.

IR $\left(\mathrm{KBr}, \mathrm{cm}^{-1}\right)(\mathbf{4 a - l}): 3252-3238,3152-$ $3147 \quad(\mathrm{NH}), \quad 1680-1677 \quad(\mathrm{C}=\mathrm{O}), \quad 1672-1669$ $(\mathrm{C}=\mathrm{O}), 1667-1665(\mathrm{C}=\mathrm{O})$.

${ }^{1} \mathrm{HNMR}(\mathbf{4 a}):\left(\mathrm{DMSO}_{\mathrm{d}}, \delta, \mathrm{ppm}\right): 1.44$ (s, $3 \mathrm{H}$, thiazol- $\left.\mathrm{C}_{5}-\mathrm{CH}_{3}\right), 1.52\left(\mathrm{~s}, 3 \mathrm{H}\right.$, thiazol- $\mathrm{C}_{5^{-}}$ $\left.\mathrm{CH}_{3}\right), 4.09\left(\mathrm{~d}, \mathrm{~J}=17.7 \mathrm{~Hz}, 1 \mathrm{H}\right.$, thiazol- $\left.\mathrm{C}_{4}-\mathrm{H}\right)$, $4.29\left(\mathrm{~m}, 2 \mathrm{H}\right.$, thiazol- $\left.\mathrm{CH}_{2}\right), 4.49(\mathrm{~s}, 2 \mathrm{H}$, $\mathrm{CH}_{2} \mathrm{NH}$ ), 6.52-7.67 (m, 12H, Ar-H), 8.50 (br s,
$1 \mathrm{H}, \mathrm{NH}, \mathrm{D}_{2} \mathrm{O}$ exchangeable), 12.22 (s, 1H, NH, $\mathrm{D}_{2} \mathrm{O}$ exchangeable).

${ }^{1} \mathrm{HNMR}$ (4b): (DMSO-d $\left.{ }_{6}, \delta, \mathrm{ppm}\right): 1.41$ (s, 3H, thiazol- $\left.\mathrm{C}_{5}-\mathrm{CH}_{3}\right), 1.44\left(\mathrm{~s}, 3 \mathrm{H}\right.$, thiazol- $\mathrm{C}_{5^{-}}$ $\left.\mathrm{CH}_{3}\right), 4.12\left(\mathrm{~d}, \mathrm{~J}=17.7 \mathrm{~Hz}, 1 \mathrm{H}\right.$, thiazol- $\left.\mathrm{C}_{4}-\mathrm{H}\right)$, $4.31\left(\mathrm{~m}, 2 \mathrm{H}\right.$, thiazol- $\left.\mathrm{CH}_{2}\right), 4.53(\mathrm{~s}, 2 \mathrm{H}$, $\mathrm{CH}_{2} \mathrm{NH}$ ), 6.52-7.71 (m, $\left.11 \mathrm{H}, \mathrm{Ar}-\mathrm{H}\right), 8.50$ (br s, $1 \mathrm{H}, \mathrm{NH}, \mathrm{D}_{2} \mathrm{O}$ exchangeable), $12.24(\mathrm{~s}, 1 \mathrm{H}, \mathrm{NH}$, $\mathrm{D}_{2} \mathrm{O}$ exchangeable).

${ }^{1} \mathrm{HNMR}$ (4c): (DMSO-d $\left.{ }_{6}, \delta, \mathrm{ppm}\right): 1.39$ (s, $3 \mathrm{H}$, thiazol- $\left.\mathrm{C}_{5}-\mathrm{CH}_{3}\right), 1.48\left(\mathrm{~s}, 3 \mathrm{H}\right.$, thiazol- $\mathrm{C}_{5^{-}}$ $\left.\mathrm{CH}_{3}\right), 4.10\left(\mathrm{~d}, \mathrm{~J}=17.7 \mathrm{~Hz}, 1 \mathrm{H}\right.$, thiazol- $\left.\mathrm{C}_{4}-\mathrm{H}\right)$, $4.32\left(\mathrm{~m}, 2 \mathrm{H}\right.$, thiazol- $\left.\mathrm{CH}_{2}\right), 4.45(\mathrm{~s}, 2 \mathrm{H}$, $\mathrm{CH}_{2} \mathrm{NH}$ ), 6.57-7.69 (m, $\left.11 \mathrm{H}, \mathrm{Ar}-\mathrm{H}\right), 8.52$ (br s, $1 \mathrm{H}, \mathrm{NH}, \mathrm{D}_{2} \mathrm{O}$ exchangeable), $12.25(\mathrm{~s}, 1 \mathrm{H}, \mathrm{NH}$, $\mathrm{D}_{2} \mathrm{O}$ exchangeable).

${ }^{1}$ HNMR (4d): (DMSO-d $\left.\mathrm{d}_{6}, \delta, \mathrm{ppm}\right): 1.43$ (s, $3 \mathrm{H}$, thiazol- $\left.\mathrm{C}_{5}-\mathrm{CH}_{3}\right), 1.48$ (s, 3H, thiazol- $\mathrm{C}_{5^{-}}$ $\left.\mathrm{CH}_{3}\right), 4.09\left(\mathrm{~d}, \mathrm{~J}=17.7 \mathrm{~Hz}, 1 \mathrm{H}\right.$, thiazol- $\left.\mathrm{C}_{4}-\mathrm{H}\right)$, $4.37\left(\mathrm{~m}, 2 \mathrm{H}\right.$, thiazol- $\left.\mathrm{CH}_{2}\right), 4.45(\mathrm{~s}, 2 \mathrm{H}$, $\mathrm{CH}_{2} \mathrm{NH}$ ), 6.57-7.72 (m, 11H, Ar-H), 8.55 (br s, $1 \mathrm{H}, \mathrm{NH}, \mathrm{D}_{2} \mathrm{O}$ exchangeable), $12.24(\mathrm{~s}, 1 \mathrm{H}, \mathrm{NH}$, $\mathrm{D}_{2} \mathrm{O}$ exchangeable). 
Table 2: Physical* and analytical data of compounds 4a-l.

\begin{tabular}{|c|c|c|c|c|c|c|c|c|c|}
\hline \multirow{2}{*}{$\begin{array}{l}\text { Comp. } \\
\text { No. }\end{array}$} & \multirow[t]{2}{*}{$\mathrm{R}^{1}$} & \multirow[t]{2}{*}{$\mathrm{R}^{2}$} & \multirow{2}{*}{$\begin{array}{c}\text { Yield } \\
(\%)\end{array}$} & \multirow{2}{*}{$\begin{array}{l}\text { Mol. Formula } \\
\text { (Mol. wt.) }\end{array}$} & \multicolumn{5}{|c|}{$\begin{array}{l}\text { Analyses (\%) } \\
\text { Calcd/Found }\end{array}$} \\
\hline & & & & & $\mathrm{C}$ & $\mathrm{H}$ & $F$ & $\mathrm{~N}$ & $S$ \\
\hline $4 a$ & $\mathrm{H}$ & $\mathrm{H}$ & 80 & $\begin{array}{c}\mathrm{C}_{26} \mathrm{H}_{25} \mathrm{~N}_{3} \mathrm{O}_{4} \mathrm{~S} \\
(475.56)\end{array}$ & $\begin{array}{c}65.67 \\
65.5\end{array}$ & $\begin{array}{c}5.30 \\
5.2\end{array}$ & ---- & $\begin{array}{c}8.84 \\
8.7\end{array}$ & $\begin{array}{c}6.74 \\
6.4\end{array}$ \\
\hline $4 b$ & $2-\mathrm{F}$ & $\mathrm{H}$ & 75 & $\begin{array}{c}\mathrm{C}_{26} \mathrm{H}_{24} \mathrm{FN}_{3} \mathrm{O}_{4} \mathrm{~S} \\
(493.55)\end{array}$ & $\begin{array}{c}63.27 \\
63.5\end{array}$ & $\begin{array}{c}4.90 \\
5.1\end{array}$ & $\begin{array}{c}3.85 \\
4.1\end{array}$ & $\begin{array}{c}8.51 \\
8.3\end{array}$ & $\begin{array}{c}6.50 \\
6.3\end{array}$ \\
\hline $4 c$ & $3-F$ & $\mathrm{H}$ & 81 & $\begin{array}{c}\mathrm{C}_{26} \mathrm{H}_{24} \mathrm{FN}_{3} \mathrm{O}_{4} \mathrm{~S} \\
(493.55)\end{array}$ & $\begin{array}{c}63.27 \\
62.9\end{array}$ & $\begin{array}{c}4.90 \\
4.7\end{array}$ & $\begin{array}{c}3.85 \\
3.6\end{array}$ & $\begin{array}{c}8.51 \\
8.4\end{array}$ & $\begin{array}{c}6.50 \\
6.2\end{array}$ \\
\hline $4 d$ & $4-F$ & $\mathrm{H}$ & 78 & $\begin{array}{c}\mathrm{C}_{26} \mathrm{H}_{24} \mathrm{FN}_{3} \mathrm{O}_{4} \mathrm{~S} \\
(493.55)\end{array}$ & $\begin{array}{c}63.27 \\
63.1\end{array}$ & $\begin{array}{c}4.90 \\
4.8\end{array}$ & $\begin{array}{c}3.85 \\
3.7\end{array}$ & $\begin{array}{c}8.51 \\
8.3\end{array}$ & $\begin{array}{c}6.50 \\
6.3\end{array}$ \\
\hline $4 e$ & $\mathrm{H}$ & 4- $\mathrm{CH}_{3}$ & 82 & $\begin{array}{c}\mathrm{C}_{27} \mathrm{H}_{27} \mathrm{~N}_{3} \mathrm{O}_{4} \mathrm{~S} \\
(489.58)\end{array}$ & $\begin{array}{c}66.24 \\
66.5\end{array}$ & $\begin{array}{c}5.56 \\
5.4\end{array}$ & ------ & $\begin{array}{c}5.58 \\
5.6\end{array}$ & $\begin{array}{c}6.55 \\
6.8\end{array}$ \\
\hline $4 f$ & $2-F$ & 4- $\mathrm{CH}_{3}$ & 86 & $\begin{array}{c}\mathrm{C}_{27} \mathrm{H}_{26} \mathrm{FN}_{3} \mathrm{O}_{4} \mathrm{~S} \\
(507.57)\end{array}$ & $\begin{array}{c}63.89 \\
63.7\end{array}$ & $\begin{array}{c}5.16 \\
5.2\end{array}$ & $\begin{array}{c}3.74 \\
3.9\end{array}$ & $\begin{array}{l}8.28 \\
8.5\end{array}$ & $\begin{array}{c}6.32 \\
6.0\end{array}$ \\
\hline $4 g$ & $3-\mathrm{F}$ & 4- $\mathrm{CH}_{3}$ & 82 & $\begin{array}{c}\mathrm{C}_{27} \mathrm{H}_{26} \mathrm{FN}_{3} \mathrm{O}_{4} \mathrm{~S} \\
(507.57)\end{array}$ & $\begin{array}{c}63.89 \\
63.5\end{array}$ & $\begin{array}{c}5.16 \\
5.4\end{array}$ & $\begin{array}{c}3.74 \\
3.5\end{array}$ & $\begin{array}{c}8.28 \\
8.5\end{array}$ & $\begin{array}{c}6.32 \\
6.1\end{array}$ \\
\hline $4 h$ & $4-F$ & 4- $\mathrm{CH}_{3}$ & 77 & $\begin{array}{c}\mathrm{C}_{27} \mathrm{H}_{26} \mathrm{FN}_{3} \mathrm{O}_{4} \mathrm{~S} \\
(507.57)\end{array}$ & $\begin{array}{c}63.89 \\
64.1\end{array}$ & $\begin{array}{c}5.16 \\
5.3\end{array}$ & $\begin{array}{c}3.74 \\
3.9\end{array}$ & $\begin{array}{c}8.28 \\
7.9\end{array}$ & $\begin{array}{c}6.32 \\
6.5\end{array}$ \\
\hline $4 i$ & $\mathrm{H}$ & 4-Cl & 74 & $\begin{array}{c}\mathrm{C}_{26} \mathrm{H}_{24} \mathrm{ClN}_{3} \mathrm{O}_{4} \mathrm{~S} \\
(510.00)\end{array}$ & $\begin{array}{c}61.23 \\
61.5\end{array}$ & $\begin{array}{c}4.74 \\
4.5\end{array}$ & ----- & $\begin{array}{c}8.24 \\
8.1\end{array}$ & $\begin{array}{c}6.29 \\
5.9\end{array}$ \\
\hline $4 \mathbf{j}$ & $2-\mathrm{F}$ & 4-Cl & 83 & $\begin{array}{c}\mathrm{C}_{26} \mathrm{H}_{23} \mathrm{ClFN}_{3} \mathrm{O}_{4} \mathrm{~S} \\
(527.99)\end{array}$ & $\begin{array}{c}59.14 \\
58.9\end{array}$ & $\begin{array}{c}4.39 \\
4.5\end{array}$ & $\begin{array}{c}3.60 \\
3.3\end{array}$ & $\begin{array}{l}7.96 \\
7.6\end{array}$ & $\begin{array}{c}6.07 \\
6.2\end{array}$ \\
\hline $4 k$ & $3-F$ & 4-Cl & 76 & $\begin{array}{c}\mathrm{C}_{26} \mathrm{H}_{23} \mathrm{ClFN}_{3} \mathrm{O}_{4} \mathrm{~S} \\
(527.99)\end{array}$ & $\begin{array}{c}59.14 \\
59.2\end{array}$ & $\begin{array}{r}4.39 \\
4.2\end{array}$ & $\begin{array}{c}3.60 \\
3.7\end{array}$ & $\begin{array}{l}7.96 \\
7.8\end{array}$ & $\begin{array}{c}6.07 \\
5.8\end{array}$ \\
\hline 41 & $4-\mathrm{F}$ & 4-Cl & 80 & $\begin{array}{c}\mathrm{C}_{26} \mathrm{H}_{23} \mathrm{ClFN}_{3} \mathrm{O}_{4} \mathrm{~S} \\
(527.99)\end{array}$ & $\begin{array}{c}59.14 \\
59.4\end{array}$ & $\begin{array}{c}4.39 \\
4.1\end{array}$ & $\begin{array}{c}3.60 \\
3.4\end{array}$ & $\begin{array}{c}7.96 \\
8.2\end{array}$ & $\begin{array}{c}6.07 \\
6.4\end{array}$ \\
\hline
\end{tabular}

*All compounds decompose before melting.

${ }^{1} \mathrm{HNMR}$ (4e): (DMSO-d $\left.\mathrm{d}_{6}, \delta, \mathrm{ppm}\right): 1.42(\mathrm{~s}$, $3 \mathrm{H}$, thiazol- $\left.\mathrm{C}_{5}-\mathrm{CH}_{3}\right), 1.53\left(\mathrm{~s}, 3 \mathrm{H}\right.$, thiazol- $\mathrm{C}_{5^{-}}$ $\left.\mathrm{CH}_{3}\right), 2.34\left(\mathrm{~s}, 3 \mathrm{H}, \mathrm{Ar}-\mathrm{CH}_{3}\right), 4.08(\mathrm{~d}, \mathrm{~J}=17.7$ $\mathrm{Hz}, 1 \mathrm{H}$, thiazol- $\left.\mathrm{C}_{4}-\mathrm{H}\right), 4.31(\mathrm{~m}, 2 \mathrm{H}$, thiazol$\left.\mathrm{CH}_{2}\right), 4.47\left(\mathrm{~s}, 2 \mathrm{H}, \mathrm{CH}_{2} \mathrm{NH}\right), 6.53-7.68(\mathrm{~m}, 11 \mathrm{H}$, Ar-H), 8.47 (br s, $1 \mathrm{H}, \mathrm{NH}, \mathrm{D}_{2} \mathrm{O}$ exchangeable), 12.62 (s, 1H, NH, $\mathrm{D}_{2} \mathrm{O}$ exchangeable).

${ }^{1} \mathrm{HNMR}$ (4f): (DMSO-d $\left.\mathrm{d}_{6}, \delta, \mathrm{ppm}\right): 1.39$ (s, $3 \mathrm{H}$, thiazol- $\left.\mathrm{C}_{5}-\mathrm{CH}_{3}\right), 1.45\left(\mathrm{~s}, 3 \mathrm{H}\right.$, thiazol- $\mathrm{C}_{5^{-}}$ $\left.\mathrm{CH}_{3}\right), 2.36\left(\mathrm{~s}, 3 \mathrm{H}, \mathrm{Ar}-\mathrm{CH}_{3}\right), 4.10(\mathrm{~d}, \mathrm{~J}=17.7$ $\mathrm{Hz}, 1 \mathrm{H}$, thiazol- $\left.\mathrm{C}_{4}-\mathrm{H}\right), 4.33$ (m, 2H, thiazol-
$\left.\mathrm{CH}_{2}\right), 4.51$ (s, 2H, $\left.\mathrm{CH}_{2} \mathrm{NH}\right), 6.55-7.73(\mathrm{~m}, 10 \mathrm{H}$, Ar-H), 8.51 (br s, $1 \mathrm{H}, \mathrm{NH}, \mathrm{D}_{2} \mathrm{O}$ exchangeable), 12.58 (s, $1 \mathrm{H}, \mathrm{NH}, \mathrm{D}_{2} \mathrm{O}$ exchangeable).

${ }^{1} \mathrm{HNMR}$ (4g): (DMSO-d $\left.{ }_{6}, \delta, \mathrm{ppm}\right): 1.40$ (s, $3 \mathrm{H}$, thiazol- $\left.\mathrm{C}_{5}-\mathrm{CH}_{3}\right), 1.47\left(\mathrm{~s}, 3 \mathrm{H}\right.$, thiazol- $\mathrm{C}_{5^{-}}$ $\left.\mathrm{CH}_{3}\right), 2.37$ (s, 3H, $\left.\mathrm{Ar}-\mathrm{CH}_{3}\right), 4.08(\mathrm{~d}, \mathrm{~J}=17.7$ $\mathrm{Hz}, 1 \mathrm{H}$, thiazol- $\left.\mathrm{C}_{4}-\mathrm{H}\right), 4.33(\mathrm{~m}, 2 \mathrm{H}$, thiazol$\left.\mathrm{CH}_{2}\right), 4.42\left(\mathrm{~s}, 2 \mathrm{H}, \mathrm{CH}_{2} \mathrm{NH}\right), 6.55-7.71(\mathrm{~m}, 10 \mathrm{H}$, Ar-H), 8.49 (br s, $1 \mathrm{H}, \mathrm{NH}, \mathrm{D}_{2} \mathrm{O}$ exchangeable), 12.65 (s, $1 \mathrm{H}, \mathrm{NH}, \mathrm{D}_{2} \mathrm{O}$ exchangeable). 
${ }^{1}$ HNMR (4h): (DMSO-d $\left.\mathrm{d}_{6}, \delta, \mathrm{ppm}\right): 1.41$ (s, 3H, thiazol- $\left.\mathrm{C}_{5}-\mathrm{CH}_{3}\right), 1.44\left(\mathrm{~s}, 3 \mathrm{H}\right.$, thiazol- $\mathrm{C}_{5^{-}}$ $\left.\mathrm{CH}_{3}\right), 2.35$ (s, 3H, Ar- $\left.\mathrm{CH}_{3}\right), 4.08(\mathrm{~d}, \mathrm{~J}=17.7$ $\mathrm{Hz}, 1 \mathrm{H}$, thiazol- $\left.\mathrm{C}_{4}-\mathrm{H}\right), 4.34(\mathrm{~m}, 2 \mathrm{H}$, thiazol$\left.\mathrm{CH}_{2}\right), 4.46\left(\mathrm{~s}, 2 \mathrm{H}, \mathrm{CH}_{2} \mathrm{NH}\right), 6.56-7.71(\mathrm{~m}, 10 \mathrm{H}$, Ar-H), 8.52 (br s, $1 \mathrm{H}, \mathrm{NH}, \mathrm{D}_{2} \mathrm{O}$ exchangeable), 12.64 (s, $1 \mathrm{H}, \mathrm{NH}, \mathrm{D}_{2} \mathrm{O}$ exchangeable).

${ }^{1} \mathrm{HNMR}(4 \mathrm{i}):\left(\mathrm{DMSO}_{\mathrm{d}}, \delta, \mathrm{ppm}\right): 1.42(\mathrm{~s}$, $3 \mathrm{H}$, thiazol- $\left.\mathrm{C}_{5}-\mathrm{CH}_{3}\right), 1.50\left(\mathrm{~s}, 3 \mathrm{H}\right.$, thiazol- $\mathrm{C}_{5}-$ $\left.\mathrm{CH}_{3}\right), 4.011\left(\mathrm{~d}, \mathrm{~J}=17.7 \mathrm{~Hz}, 1 \mathrm{H}\right.$, thiazol- $\left.\mathrm{C}_{4}-\mathrm{H}\right)$, $4.30\left(\mathrm{~m}, 2 \mathrm{H}\right.$, thiazol- $\left.\mathrm{CH}_{2}\right), 4.47(\mathrm{~s}, 2 \mathrm{H}$, $\mathrm{CH}_{2} \mathrm{NH}$ ), 6.53-7.69 (m, $\left.11 \mathrm{H}, \mathrm{Ar}-\mathrm{H}\right), 8.51$ (br s, $1 \mathrm{H}, \mathrm{NH}, \mathrm{D}_{2} \mathrm{O}$ exchangeable), $12.82(\mathrm{~s}, 1 \mathrm{H}, \mathrm{NH}$, $\mathrm{D}_{2} \mathrm{O}$ exchangeable).

${ }^{1} \mathrm{HNMR}(\mathbf{4 j})$ : (DMSO-d $\left.\mathrm{D}_{6}, \delta, \mathrm{ppm}\right): 1.42(\mathrm{~s}$, $3 \mathrm{H}$, thiazol- $\left.\mathrm{C}_{5}-\mathrm{CH}_{3}\right), 1.46\left(\mathrm{~s}, 3 \mathrm{H}\right.$, thiazol- $\mathrm{C}_{5^{-}}$ $\left.\mathrm{CH}_{3}\right), 4.10\left(\mathrm{~d}, \mathrm{~J}=17.7 \mathrm{~Hz}, 1 \mathrm{H}\right.$, thiazol- $\left.\mathrm{C}_{4}-\mathrm{H}\right)$, $4.32\left(\mathrm{~m}, 2 \mathrm{H}\right.$, thiazol $\left.-\mathrm{CH}_{2}\right), 4.51(\mathrm{~s}, 2 \mathrm{H}$, $\mathrm{CH}_{2} \mathrm{NH}$ ), 6.55-7.73 (m, 10H, Ar-H), 8.47 (br s, $1 \mathrm{H}, \mathrm{NH}, \mathrm{D}_{2} \mathrm{O}$ exchangeable), $12.82(\mathrm{~s}, 1 \mathrm{H}, \mathrm{NH}$, $\mathrm{D}_{2} \mathrm{O}$ exchangeable).

${ }^{1}$ HNMR (4k): (DMSO-d $\left.\mathrm{d}_{6}, \delta, \mathrm{ppm}\right): 1.40$ $\left(\mathrm{s}, 3 \mathrm{H}\right.$, thiazol $\left.-\mathrm{C}_{5}-\mathrm{CH}_{3}\right), 1.44\left(\mathrm{~s}, 3 \mathrm{H}\right.$, thiazol- $\mathrm{C}_{5^{-}}$ $\left.\mathrm{CH}_{3}\right), 4.08\left(\mathrm{~d}, \mathrm{~J}=17.7 \mathrm{~Hz}, 1 \mathrm{H}\right.$, thiazol- $\left.\mathrm{C}_{4}-\mathrm{H}\right)$, $4.34\left(\mathrm{~m}, 2 \mathrm{H}\right.$, thiazol- $\left.\mathrm{CH}_{2}\right), 4.46(\mathrm{~s}, 2 \mathrm{H}$, $\mathrm{CH}_{2} \mathrm{NH}$ ), 6.56-7.74 (m, 10H, Ar-H), 8.51 (br s, $1 \mathrm{H}, \mathrm{NH}, \mathrm{D}_{2} \mathrm{O}$ exchangeable), $12.78(\mathrm{~s}, 1 \mathrm{H}, \mathrm{NH}$, $\mathrm{D}_{2} \mathrm{O}$ exchangeable).

${ }^{1} \mathrm{HNMR}$ (4l): (DMSO-d $\left.\mathrm{d}_{6}, \delta, \mathrm{ppm}\right): 1.41$ (s, $3 \mathrm{H}$, thiazol- $\left.\mathrm{C}_{5}-\mathrm{CH}_{3}\right), 1.45$ (s, $3 \mathrm{H}$, thiazol- $\mathrm{C}_{5^{-}}$ $\left.\mathrm{CH}_{3}\right), 4.08\left(\mathrm{~d}, \mathrm{~J}=17.7 \mathrm{~Hz}, 1 \mathrm{H}\right.$, thiazol- $\left.\mathrm{C}_{4}-\mathrm{H}\right)$, $4.35\left(\mathrm{~m}, 2 \mathrm{H}\right.$, thiazol- $\left.\mathrm{CH}_{2}\right), 4.42(\mathrm{~s}, 2 \mathrm{H}$, $\mathrm{CH}_{2} \mathrm{NH}$ ), 6.54-7.70 (m, 10H, Ar-H), 8.53 (br s, $1 \mathrm{H}, \mathrm{NH}, \mathrm{D}_{2} \mathrm{O}$ exchangeable), $12.84(\mathrm{~s}, 1 \mathrm{H}, \mathrm{NH}$, $\mathrm{D}_{2} \mathrm{O}$ exchangeable).

\section{Antimicrobial activity}

The microdilution susceptibility test in Müller-Hinton Broth (Oxoid) and Sabouraud Liquid Medium (Oxoid) were used for the determination of antibacterial and antifungal activity. ${ }^{13}$ Test organisms are Escherichia coli (E. coli) ATCC 25922 as Gram-negative bacteria, Staphylococcus aureus (S. aureus) ATCC 19433 as Gram-positive bacteria and Candida albicans (C. albicans) as yeast like fungi. Ampicillin trihydrate and clotrimazole were used as standard antibacterial and antifungal agents respectively. Solutions of the test compounds, ampicillin trihydrate and clotrimazole were prepared in DMSO at concentration of $1600 \mu \mathrm{g} / \mathrm{ml}$. The test compounds were two-fold serially diluted (800,
$400, \ldots . .6 .25 \mu \mathrm{g} / \mathrm{ml})$. To each well, equal volume of double strength broth previously inoculated with standardized inoculum of the three test organisms $(1 \mathrm{x} 106 \mathrm{CFU} / \mathrm{ml})$ (Colony Forming Unit $/ \mathrm{ml}$ ) was added. The inoculated plates were incubated at $36^{\circ}$. The MIC values were determined after $24 \mathrm{~h}$. Table 3.

Table 3: Minimal inhibitory concentrations (MIC, $\mu \mathrm{g} / \mathrm{ml}$ ) of test compounds.

\begin{tabular}{|c|c|c|c||}
\hline $\begin{array}{c}\text { Test } \\
\text { compound }\end{array}$ & $\begin{array}{c}E . \\
\text { coli }\end{array}$ & $\begin{array}{c}\text { S. } \\
\text { aureus }\end{array}$ & $\begin{array}{c}\text { C. } \\
\text { albicans }\end{array}$ \\
\hline $\mathbf{4 a}$ & 12.5 & 25 & $>200$ \\
\hline $\mathbf{4 b}$ & 25 & 50 & $>200$ \\
\hline $\mathbf{4 c}$ & 25 & 25 & $>200$ \\
\hline $\mathbf{4 d}$ & 12.5 & 12.5 & $>200$ \\
\hline $\mathbf{4 e}$ & 50 & 50 & $>200$ \\
\hline $\mathbf{4 f}$ & 50 & 25 & $>200$ \\
\hline $\mathbf{4 g}$ & 50 & 25 & $>200$ \\
\hline $\mathbf{4 h}$ & 12.5 & 12.5 & $>200$ \\
\hline $\mathbf{4 i}$ & 100 & 100 & $>200$ \\
\hline $\mathbf{4 j}$ & 50 & 100 & $>200$ \\
\hline $\mathbf{4 k}$ & 50 & 50 & $>200$ \\
\hline $\mathbf{4 l}$ & 12.5 & 12.5 & $>200$ \\
\hline Ampicillin & 25 & 12.5 & --- \\
\hline Clotrimazole & --- & --- & 12.5 \\
\hline \hline
\end{tabular}

\section{RESULTS AND DISCUSSION}

\section{Chemistry}

The target compounds were synthesized through the reaction sequence shown in Scheme 1. The selected thiazolidine derivatives were obtained by deprotection of N-Boc substituted thiazolidines 1a-c using $4 \mathrm{~N}$ $\mathrm{HCl} /$ Dioxane. Condensation of 2,5-dihydroxybenzoic acid (gentisic acid) with the appropriate thiazolidine derivative was performed using 1-ethyl-3-(3-dimethyl aminopropyl) carbodiimide hydrochloride (EDC. $\mathrm{HCl})$ and hydroxybenzotriazole (HOBt). EDC.HCl was used as a water soluble carbodiimide to form an active ester between gentisic acid and hydroxybenzotriazole. The so formed active ester can easily coupled with the $\mathrm{N}$-deprotected thiazolidine derivative to afford the (4R)-3-(2,5-dihydroxybenzoyl)-5,5dimethyl-N-substituted benzylthiazolidine-4carboxamide 2a-d in good yields. The latter 
<smiles>CC(C)(C)OC(=O)N1CSC(C)(C)[C@H]1C(=O)NCc1ccc[pH]1</smiles>

1a-d

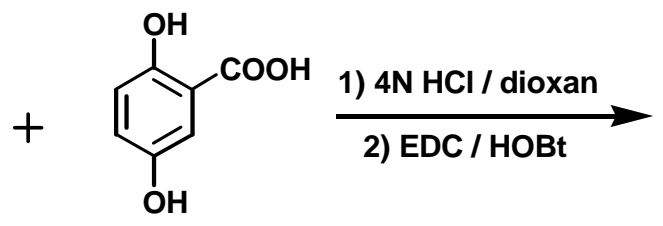

2) EDC / HOBt<smiles>[H][Y]1cccc(CNC(=O)[C@@H]2N(C(=O)c3cc(O)ccc3O)CSC2(C)C)c1</smiles><smiles>CC1(C)SCN(C(=O)C2=CC(=O)C=CC2=O)C1C(=O)NCc1ccccc1</smiles>

$$
\begin{aligned}
& \mathbf{R}^{1}=\mathrm{H}, 2-\mathrm{F}, 3-\mathrm{F}, 4-\mathrm{F} \\
& \mathbf{R}^{2}=\mathrm{H}, \mathrm{CH}_{3} \mathrm{Cl}
\end{aligned}
$$

\section{Scheme 1}

compounds were oxidized with ferric chloride to the corresponding 1,4-benzoquinones 3a-d. The reaction between 1,4-benzoquinone derivatives 3a-d and aniline or 4-substituted aniline resulted in the sought for 2,5disubstituted-1,4-benzoquinone derivatives 4al., Tables $1 \& 2$.

\section{Antimicrobial activity}

The designed compounds 4a-l were evaluated for their antimicrobial activity. The microdilution susceptibility test in MüllerHinton Broth (Oxoid) and Sabouraud Liquid Medium (Oxoid) was used for the determination of antibacterial and antifungal activity respectively. The minimal inhibitory concentrations (MICs) listed in Table 3 showed that all test compounds were inactive against $C$. albicans and showed higher activity against $E$. coli than $S$. aureus. The activity of compounds $\mathbf{4 a}, \mathbf{4 d}, \mathbf{4 h}$ and $\mathbf{4 l}$ was double that of ampicillin against E. coli While compounds $\mathbf{4 b}$ and $\mathbf{4 c}$ were equipotent to ampicillin against the same organism. Other compound showed 50\% activity or less than that of ampicillin, while compounds $\mathbf{4 d}, \mathbf{4 h}$ and $4 \mathrm{l}$ were equipotent to ampicillin against $S$. aureus. In addition, the activity of $\mathbf{4 a}, \mathbf{4 c}, \mathbf{4 f}$ and $\mathbf{4 g}$ was $50 \%$ of that of ampicillin against $S$. aureus, whereas other compounds possessed activity against $S$. aureus less than $50 \%$ of that of ampicillin. It is worthmentioning that, the flouro derivatives showed a pronounced antibacterial activity in this study.

It could be safely concluded that introduction of fluorine atom in the parapostion of the phenyl group attached to the thiazolidine ring system of the novel compounds, resulted in the most active compounds against E. coli and S. aureus (4d, 4h and 4l). 


\section{REFERENCES}

1- R. Finch, Medicine, 1, 27-30 (1996).

2- N. H. Georgopapadakou and T. J. Walsh, Antimicrob. Agents Chemother., 40, 279291 (1996).

3- E. Mutschler and H. Derendorf, "Drug Actions - Basic Principles and Therapeutic Aspects", Medpharm Scientific Publishers, Stuttgart (1995).

4- A. A. Bekhit, I. Chaaban and Y. S. AbdelGhany, "Proceedings of Assiut University $2^{\text {nd }}$ Pharmaceutical Sciences Conference", 8-9 March, 2000, pp.107-114.

5- I. Chaaban, A. A. Bekhit and Y. S. AbdelGhany, Egypt. J. Pharm. Sci., 39, 91-107 (1998).

6- M. A. Hassan, A. O. Maslat, M. Abussaud, I. C. Ahmed and A. S. AlKofahi, Arch. Pharm. Pharm. Med. Chem., 331, 385-338 (1998).

7- I. Chaaban, E. M. El-Khawass, O. M. Aboulwafa and A. Hazzaa, Acta. Pharm. Jugoslav., 39, 143-149 (1989).
8- A. M. Farghaly, A. A. Bekhit and J. Y. Park, Arch. Pharm. Pharm. Med. Chem., 333, 53-57 (2000).

9- A. A. Bekhit, H. Matsumoto, H. M. M. Abdel-Rahman, T. Mimoto, S. Nojima, H. Takaku, T. Kimura, K. Akaji, Y. Kiso and Y. Shimonishi (ed): "Peptide Science Present and Future", 1999, Kluwer Academic Publisher, Great Britain, pp. 660-661.

10- I. M. Roushdi, A. A. Mikhail and I. Chaaban, Acta. Pharm. Jugoslav., 26, 277 285 (1976).

11- M. Z. Barakat, S. K. Shehab and M. M. El-Sadr, J. Chem. Soc., 901-902 (1958).

12- F. I. Carrol, K. H. Dudley and H. W. Miller, J. Med. Chem., 12, 187-189 (1969).

13- P. R. Murray, E. J. Baron, M. A. Pfaller, F. C. Tenover, R. H. Yolken, "Manual of Clinical Microbiology", Woods G. L. and Washington J. A. (Eds), "Antimicrobial Agents and Susceptibility Testing", Am. Soc. Microbiol., Washington, DC (1995). 